\title{
On the Delay and Data Rate Performance of Throughput-Efficient Multi-Channel Access Algorithms in Cognitive Radio Networks
}

\author{
Luca Zappaterra, Hyeong-Ah Choi \\ Department of Computer Science \\ George Washington University \\ Washington DC, USA \\ Email: \{lucaz, hchoi\}@gwu.edu
}

\author{
Kamran Sayrafian-Pour \\ Information Technology Laboratory \\ National Institute of Standards and Technology \\ Gaithersburg MD, USA \\ Email: ksayrafian@nist.gov
}

\author{
Jari H. Iinatti \\ Centre for Wireless Communications \\ University of Oulu \\ Oulu, Finland \\ Email: jari.iinatti@ee.oulu.fi
}

\begin{abstract}
Cognitive Radio Networks (CRNs) are designed to increase spectrum utilization by allowing unlicensed secondary users (SUs) to operate over existing wireless channels where primary users (PUs) have license to transmit. An SU searches for channels not currently occupied by PUs, it estimates their link quality and it aggregates the resources found available for transmission, by means of multi-channel access. Typically, optimal stopping algorithms decide when to interrupt the exploration phase and start exploiting the channels for transmission, with the goal of throughput maximization.

In this paper we recognize the importance of evaluating the performance of throughput-efficient multi-channel access algorithms in terms of delay and data rate, which constitute fundamental traffic requirements for mobile applications. Through extensive simulations, we have derived guidelines for supporting SUs with stringent traffic requirements of delay, jitter and guaranteed data rate in the current multi-channel algorithms under multiple CRN scenarios. The encouraging results of the conducted performance evaluation motivate our interest in further researching multi-channel access algorithms for the optimization of application-specific requirements.
\end{abstract}

\section{INTRODUCTION}

The wireless spectrum has been historically assigned with redundancy to single applications which often utilize their frequencies for very limited portions of space and in restricted areas [1]. This has led to the spectrum scarcity problem, where there may not exist available frequencies to assign for emerging wireless applications soon.

Cognitive radio networks (CRNs) represent the most promising approach to ease the spectrum scarcity problem. CRNs target at increasing the utilization of the wireless channels by allowing unlicensed secondary users (SUs) to opportunistically access the parts of the spectrum (i.e., spectrum holes) left available by licensed primary users (PUs), without disruptively interfering with their activity.

The recent release of the first CRN-based IEEE Standard 802.22 [2] and the ongoing government regulatory plans for spectrum allocations have pushed the interest towards enabling CRN functionalities in the design of current and future wireless applications. Examples include public safety networks, cellular networks, smart grids, body area networks and ad-hoc networks [3].

A significant challenge for the successful applicability of CRN techniques to mobile networks is represented by the difficulty of guaranteeing Quality-of-Service (QoS) support to SUs in such an opportunistic transmission environment. The first problem comes from the fact that SUs need to release the resources to the PUs within a short interval, when the latter claim them back. Secondly, SUs need to explore the CRN channels, to find availability and estimate the quality of channels. This process is typically modeled as an optimal stopping problem [4], which may incur a significant channel access delay.

Multi-channel access algorithms for CRNs target at finding the optimal set of available channels to be exploited simultaneously for transmission by means of channel aggregation and channel bonding techniques enabled by the OFDM technology [5], [6]. Previous works such as [7]-[10] including ours [11], [12], formulate the decision on when to stop the exploration phase and transmit as an optimal stopping problem, oriented to maximize the throughput of SUs. Particularly, in [12] we have developed an efficient and exact algorithm that finds the optimal set of channels for transmission, with low algorithmic complexity, making it feasible for resource constrained devices such as mobile SUs, where other solutions would fail because of the elevate algorithmic complexity.

By evaluating QoS traffic requirements such as delay and data rate and their variations in our throughput-efficient multichannel access algorithms, we have identified conditions that satisfy the SU traffic requirements of stable channel access delay and data rate, typical of mobile applications. We have derived insights on how these conditions are strongly dependent on the particular and possibly variable characteristics of the CRN considered, such as number of channels, PU activity, sensing and channel estimation delays, and available transmission rates. Therefore, we foresee a great benefit in studying and developing multi-channel access algorithms based on the optimal stopping theory, that will allow SUs to make network decisions with the goal of optimizing their 
specific traffic requirements such as delay, jitter, or guaranteed data rate. Future research on these algorithms could enable the successful operation of different mobile applications as SUs in a CRN.

The rest of the paper is organized as follows. Section II explains the system model and the details of the algorithms evaluated. Section III compares the performance of the considered algorithms in varied CRN scenarios. Finally, section IV concludes the paper.

\section{PReliminaries}

The contributions of this paper extend the findings of [11] and [12]. Here we revisit the system model adopted and the key aspects of the algorithms proposed.

\section{A. System model}

A CRN operates on a set of channels $\mathbf{C}=\{1,2, \ldots, N\}$. A time-slotted model is considered, which is motivated by the following observation: in order to protect their operations, PUs specify a maximum interference tolerance period $T$, which prevents SUs' disruptive transmission, not allowing them to transmit on a channel during $T$ if PU activity is sensed on it. In the adopted system, the PU activity on each channel is either present or not during the entire duration of the transmission slot $T$. The first part of each interval $T$ is dedicated to channel exploration, during which an SU searches for channels to use for transmission; the remaining part of the interval $T$ is called channel exploitation and used for data transmission [13].

Since we are interested in characterizing the performance of mobile SUs, we assume devices are equipped with only one transceiver, therefore only able to either sense or transmit on a channel at the same time. We consider channel switchingtime negligible. The channel exploration phase is divided into one or more steps of duration $\tau$. At each step, an SU wanting to communicate with another SU, firstly senses the possible presence of PU activity on a channel, then it estimates the link quality between the two nodes. The sensing process returns the state of a channel (i.e., available or busy) in respect to PU occupancy, by using existing techniques such as energy detection [14] and feature detection [15]. Error-free sensing is assumed for simplicity. After finding a channel available, channel estimation evaluates the link quality, which translates into supported data rate, between the communicating SUs, accomplished by transmitting and feeding back a training sequence between the SU-transmitter and the SU-receiver.

A sequence $\mathbf{S}_{\mathbf{M}}=\left\langle s_{1}, \ldots, s_{M}\right\rangle$, with $M \leqslant N$, specifies the channel exploration order, with $s_{m}$ being the channel sensed and estimated at the $m$-th step. For generality, we assume random $\mathbf{S}_{\mathbf{M}}$, pointing at [8], [9], [16] for more details on the implications of the channel exploration order. After exploring channel $s_{m}$, an SU decides if extending the exploration phase to the step $m+1$, because the achievable throughput guaranteed by the channels discovered so far can be improved by the next channels, or stop the channel exploration phase, if the additional time lost in sensing and estimating
TABLE I

SUMMARY OF RELEVANT SYMBOLS

\begin{tabular}{|c||c|}
\hline Symbol & Description \\
\hline \hline $\mathbf{C}$ & Set of channels in the CRN \\
$\mathbf{P}_{\mathbf{c}}$ & Probability distribution of data rates for channel $c$ \\
$\mathbf{R}$ & Set of supported data rates \\
$\mathbf{S}_{\mathbf{M}}$ & Channel exploration order sequence \\
$A_{m}$ & Attainable data rate after exploring channels $s_{1}, \ldots, s_{m}$ \\
$K$ & Number of data rates supported \\
$M$ & Maximum number of explorable channels during $T$ \\
$N$ & Number of channels in the CRN \\
$T$ & Transmission slot duration \\
$Y_{m}$ & Throughput in $T$, after exploring channels $s_{1}, \ldots, s_{m}$ \\
$a_{c}$ & Attainable data rate of channel $c$ \\
$p_{c, k}$ & Probability of obtaining rate $r_{k}$ for channel $c$ \\
$q_{c}$ & Availability probability of channel $c$ \\
$r_{k}$ & $k$-th supported data rate value \\
$s_{m}$ & $m$-th explored channel \\
$\tau$ & Channel exploration step time \\
\hline
\end{tabular}

cancels the benefit of finding new resources to be aggregated for transmission.

We define $Y_{m}=A_{m} \cdot h_{m}$ as the throughput attainable with the aggregate data rate $A_{m}=\sum_{j=1}^{m} a_{s_{j}}$, obtainable adding the rates $a_{s_{j}}$ of the channels found available among $s_{1}, \ldots, s_{m}$ (note that $a_{s_{j}}=0$ if channel $j$ has PU activity present on it). $h_{m}=\max \{(T-m \tau) / T, 0\}$ is a scaling factor. As in [10], [11], [17], our model assumes each SU has the following CRN statistics available: an array of channel availability probabilities $\mathbf{Q}=\left[q_{1}, q_{2}, \ldots, q_{N}\right]$, which models PU activity on each channel in $\mathbf{C}$; the probability distribution $\mathbf{P}_{\mathbf{c}}=\left[p_{c, 1}, p_{c, 2}, \ldots, p_{c, K}\right]$ for the supported data rates $\mathbf{R}=\left[r_{1}, r_{2}, \ldots, r_{K}\right]$ (with $r_{1}<r_{2}<\ldots<r_{K}$ ) for each channel $c$. These statistics are used to aid the decision on when to terminate channel exploration phase and initiate the data transmission. As a reference, a list of relevant symbols used throughout this paper is summarized in Table I.

\section{B. Multi-channel access algorithms}

The Backward Induction algorithm defined in [11] finds the optimal stopping time that maximizes the expected throughput during $T$ by defining $V_{m, A_{m}}$ as the the optimal expected maximum throughput when $m$ channels have been explored and the attainable aggregate data rate at the $m$-th step is $A_{m}$, which is derived through the following dynamic programming recurrence relation:

$$
\begin{aligned}
V_{M, l} & =\left.Y_{M}\right|_{A_{M}=l} \quad \forall l \in \Psi_{R, M} \\
V_{m, l} & =\max \left\{\left.Y_{m}\right|_{A_{m}=l},\left(1-q_{s_{m+1}}\right) V_{m+1, l}+\right. \\
+ & \left.q_{s_{m+1}} \sum_{k=1}^{K} p_{s_{m+1}, k} V_{m+1, l+r_{k}}\right\} \\
& \forall l \in \Psi_{R, m} \text { and } 1 \leqslant m<M
\end{aligned}
$$


$\left.Y_{m}\right|_{A_{m}=l}$ represents the attainable throughput at step $m$, given $l$ aggregate data rate has been discovered. $\Psi_{R, m}$ is the set of possible unique aggregate data rate values obtainable from discovering $m$ channels with supported rates $\mathbf{R}$.

As an attempt to reduce the offline complexity for precomputing the rewards' matrix $\mathbf{V}$, which has asymptotic complexity of $O\left(M^{K}\right)$, we have defined the value $W_{m, A_{m}}$, which at every channel exploration step $m$ computes the largest expected aggregate throughput that can be possibly achieved by extending the exploration phase to channels from $s_{m+1}$ to $s_{M}$, defined as:

$$
W_{m, A_{m}}=\max _{m<n \leqslant M} W_{m, A_{m}}^{(n)}=\max _{m<n \leqslant M}\left\{\left(A_{m}+B_{m}^{(n)}\right) h_{n}\right\}
$$

where

$$
B_{m}^{(n)}=\sum_{j=m+1}^{n} D_{s_{j}}=\sum_{j=m+1}^{n} q_{s_{j}} \sum_{k=1}^{K} r_{k} p_{s_{j}, k}
$$

represents the additional aggregate data rate expected by exploring the channels $s_{m+1}$ to $s_{n}$ and $D_{s_{j}}$ is the expected data rate of channel $s_{j}$.

Starting from Equation 3, which suggests that an SU should stop channel exploration and transmit when $Y_{m} \geqslant W_{m, A_{m}}$, we have demonstrated the following necessary and sufficient condition:

$$
A_{m} \geqslant \delta_{m}^{(n)} \Longleftrightarrow Y_{m} \geqslant W_{m, A_{m}}^{(n)} \quad \forall m, n, 1 \leqslant m<n \leqslant M
$$

where

$$
\delta_{m}^{(n)}=\frac{B_{m}^{(n)} h_{n} T}{(n-m) \tau} \quad \forall n, m<n \leq M
$$

The values $\delta=\left\{\delta_{1}, \ldots, \delta_{M-1}\right\}$, where $\delta_{m}=$ $\max _{m<n \leqslant M}\left\{\delta_{m}^{(n)}\right\}$, and $\boldsymbol{\beta}=\left\{\beta_{0}, \beta_{1}, \ldots, \beta_{M}\right\}$ with $\beta_{0}=0$ and $\beta_{j}=\beta_{j-1}+D_{s_{j}}$, for $1 \leqslant j \leqslant M$ can be precomputed offline. Then, at each exploration step $m$, given $B_{m}^{(n)}=\beta_{n}-\beta_{m}$, the stopping rule simply requires to stop exploring channels and transmit when $A_{m} \geqslant \delta_{m}$. This solution is called Low-Complexity algorithm, since it only needs $O\left(M^{2}\right)$ and $O(M)$ in the offline and online phases respectively.

\section{Performance Evaluation}

In this section we have studied the performance of the proposed algorithms in terms of channel access delay and data rate and their variations, for the considered network. These two metrics give us an estimate of the feasibility of our solutions for applications with the stringent traffic requirements typical of mobile SUs and low-power SUs.

For absolute performance evaluation, we have considered an optimum solution, called Oracle algorithm, which knows at the beginning of each interval $T$ the attainable data rate $a_{s_{m}}$ of each channel $s_{m}$. SUs still need to go through the channel exploration steps, but the optimum stopping time is guaranteed.
We have used computer simulations to evaluate the performance of our algorithms in terms of data rate and channel access delay and their variability under different CRN scenarios. In all the experiments the following assumptions are made: 1) all $N$ channels in the CRN can be potentially evaluated during the same channel exploration phase within $T$ (i.e., $M=N$ ); 2) on each channel, a PU-transmitter generates burst sessions of duration multiple of $T$, destined to a PU-receiver operating on the same channel, with inter-arrival times generated according to a Poisson process with varying parameter $\mu$, and service times exponentially distributed with parameter $\lambda=0.1$.

We have conducted two sets of experiments: a thorough study of channel access delay and data rate metrics under multiple CRN scenarios; the evaluation of channel access delay for SUs with only single-rate transmission capability.

\section{A. Channel access delay and data rate evaluation}

Two types of mobile CRNs are considered: in the first type the number of channels is $M=20$ and SUs have faster sensing and channel estimation capabilities that can identify PU activity and the maximum supported data rate on a channel with channel exploration step durations of $\tau=400 \mu \mathrm{s}$; the second type of CRN has $M=4$ channels, with SUs needing a longer interval $\tau=2 \mathrm{~ms}$ to sense and estimate channel quality for each explored channel. In both cases $T=10 \mathrm{~ms}$ and the maximum possible channel exploration phase duration does not occupy more than $80 \%$ of the whole transmission slot $T$. Five data rates (i.e., $R=[1,2,3,4,5] \mathrm{Mb} / \mathrm{s}$ ) are supported, a reasonable assumption with current PHY technologies. It is important to notice how the units of measurement chosen do not limit the validity of our results.

Three PU activity scenarios with varying mean channel availability probability $q$ are considered : high activity $(q=$ $0.25)$, medium activity $(q=0.5)$, and low activity $(q=0.75)$. For each PU activity scenario, four different cases, characterized by varying PU activity and link qualities are studied:

- Uniform rates scenario has uniform distribution of supported data rates in all channels and homogeneous PU activity among channels.

- Heterogeneous channels considers heterogeneous PU activity among channels, with four types of PU activity distributed equally among the $N$ channels: $q^{\prime}=q-0.15$, $q^{\prime \prime}=q-0.05, q^{\prime \prime \prime}=q+0.05$, and $q^{\prime \prime \prime \prime}=q+0.15$; supported data rate values are uniformly distributed.

- Scarce quality scenario has $75 \%$ of channels of type $c_{1}$ with higher probabilities of lower data rates (i.e., $\left.\mathbf{P}_{\mathbf{c}_{1}}=[0.5,0.3,0.2,0,0]\right)$, and $25 \%$ of type $c_{2}$ with higher probabilities of higher data rates (i.e., $\mathbf{P}_{\mathbf{c}_{\mathbf{2}}}=$ $[0,0,0.2,0.3,0.5])$; homogeneous PU activity among channels is assumed.

- Good quality scenario has $75 \%$ of channels of type $c_{2}$ with higher probabilities of higher data rates, and $25 \%$ channels of type $c_{1}$ with higher probabilities of lower data rates; homogeneous PU activity among channels is assumed. 


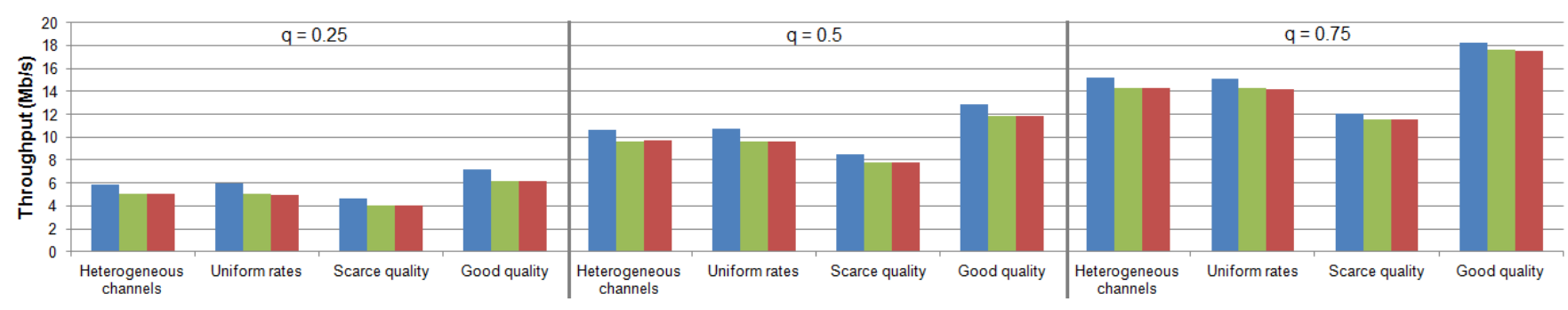

(a)

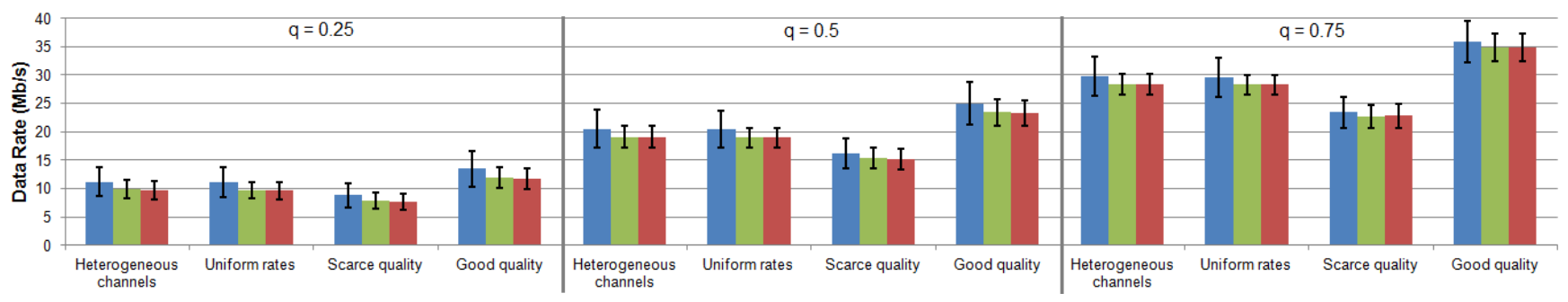

(b)

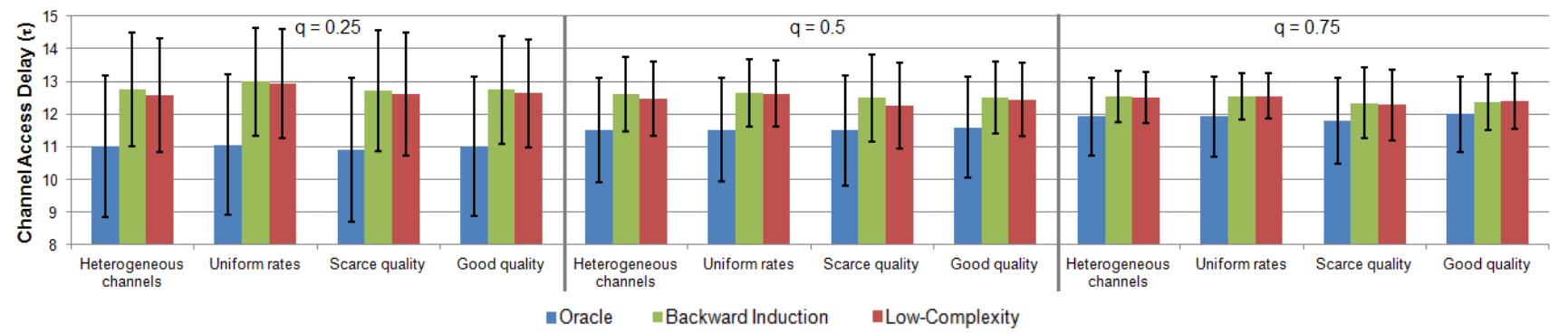

Fig. 1. Mean throughput (a), data rate mean and standard error (b), and channel access delay mean and standard error (c) for the studied algorithms under multiple link quality and PU activity scenarios, with $M=20$.

Figure 1 shows the simulation results for the three algorithms considered for mean data rate with its standard error and mean channel access delay with its standard error, for the cases described when $N=20$. The throughput values are reported as a reference to show the equivalency of the Backward Induction and Low-Complexity algorithms and their proximity with the Oracle performance.

Looking at the set of experiments as a whole, the collected data shows the Low-Complexity and Backward induction algorithm achieving almost identical values for throughput but in different ways: the Low-Complexity algorithm tends to be more conservative, by stopping the channel exploration earlier, therefore obtaining shorter channel access times than the Backward Induction solution. This is a very interesting result that shows our algorithm being efficient for applications where channel access delay is critical. In all the considered scenarios the variation of both data rates and channel access delays for the Low-Complexity and Backward induction are very similar and significantly less than the Oracle variations. This indicates the validity of the algorithms for delay and data rate sensitive SU applications.

Figure 2 shows the same set of results of Figure 1 for $M=$ 4. The former observation of the Low-Complexity algorithm having shorter delays than the Backward induction algorithm does not hold anymore. Additionally, both proposed algorithms show significant standard error values in both data rates and channel access delays. The instability of these metrics makes the operation of SUs with delay and data rate sensitive traffic challenging in SUs with limited number of channels.

\section{B. Channel access delay for single-rate SUs}

For this experiment, we consider the specific case of SUdevices, such as low-power devices, that cannot perform channel estimation and therefore they transmit using multichannel access with minimum supported data rate of $r=1$ on each used channel. In this way, the channel exploration phase reduces to only sensing the PU presence on each channel explored. Again keeping the same maximum possible channel exploration phase duration to $80 \%$ of $T$, we evaluate the mean channel access delay experienced by SUs under varying homogeneous PU activity occupations among channels, i.e, varying $q=q_{s_{m}}, \forall m, 1 \leqslant m \leqslant M$. Three cases representing different CRNs are considered: $M=4, M=8$, and $M=16$.

Figure 3 shows the mean channel access delay for the LowComplexity and Oracle solutions under varying PU activity (i.e., q). For all the $M$ cases, the Low-Complexity values are very close to the optimum throughput solution, with differences of at most $1.3 \tau$ for the case of $M=16$. For lower values of $M$, the Low-Complexity algorithm provides even shorter delays than the Oracle solution: for $M=4$ 

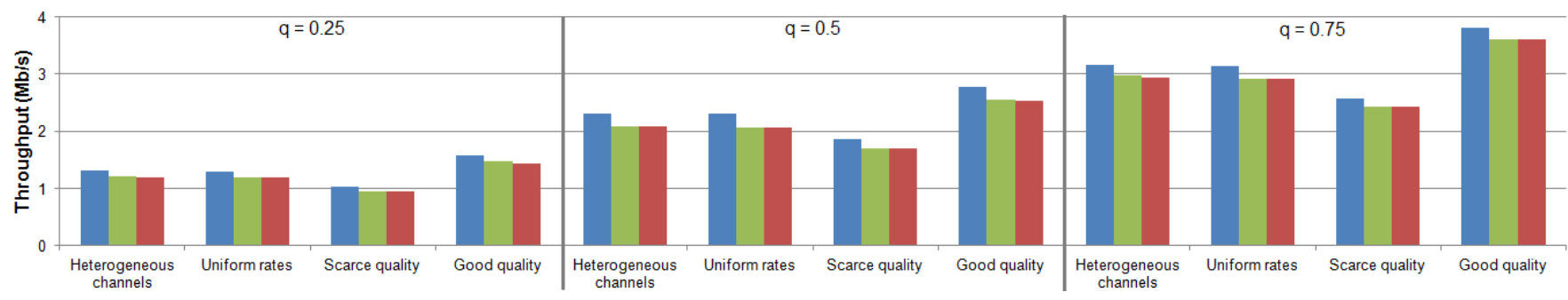

(a)

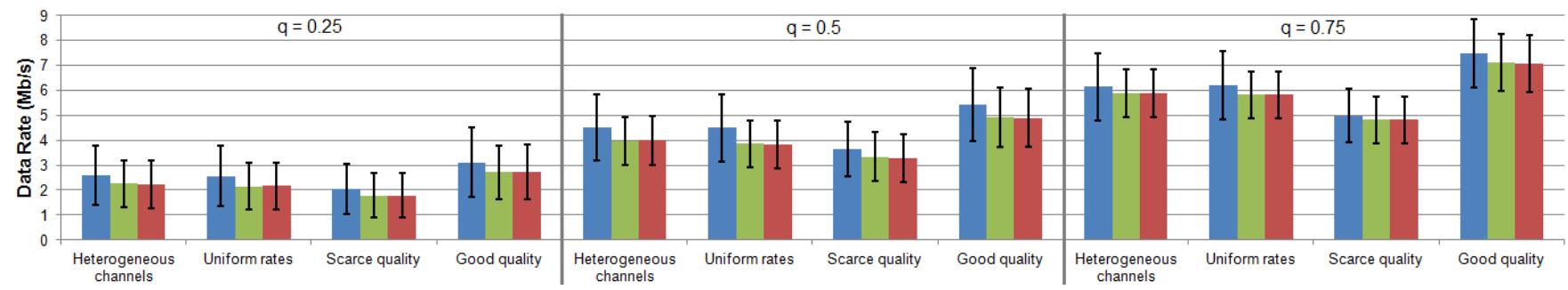

(b)

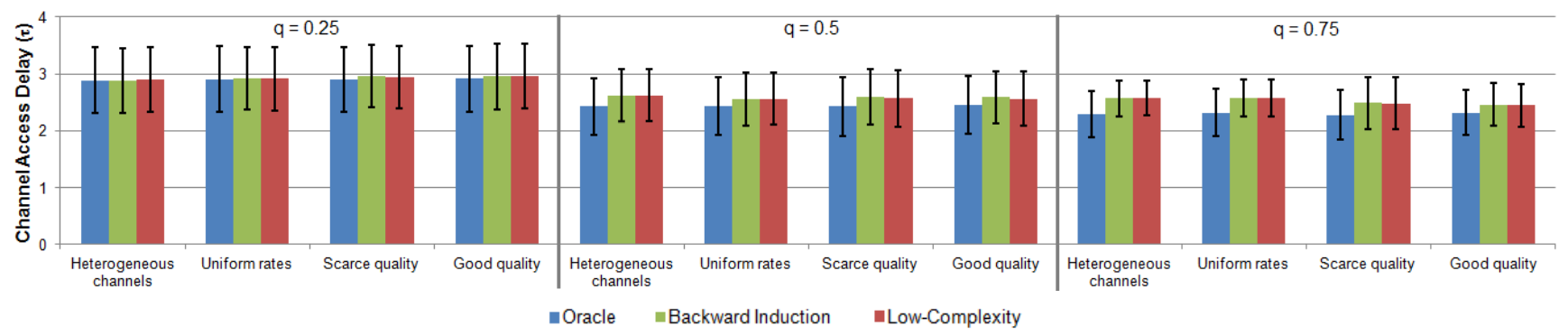

Fig. 2. Mean throughput (a), data rate mean and standard error (b), and channel access delay mean and standard error (c) for the studied algorithms under multiple link quality and PU activity scenarios, with $M=4$.

and $M=8$ when channel availability values are less than 0.35 and 0.18 respectively. This happens because the LowComplexity algorithm has a more conservative approach than the Oracle during channel exploration. In this way, a modest amount of throughput is scarified by the Low-Complexity algorithm, as demonstrated in [12], but reducing the average time an SU has to wait to access the aggregate channels that provide the optimal throughput. With average channel access delays varying at most of $1.25 \tau, 0.85 \tau$, and $0.8 \tau$ respectively for the increasing values of $\mathrm{M}$ considered, the coarse-grained stability of the Low-Complexity case suggests the feasibility of our solution for delay-sensitive application, independently of the PU activity present in the CRN. On another side, the sawtooth shape of the Low-Complexity curves highlights a fine-grained instability of delay values, with abrupt changes happening for certain configurations of PU activity. Therefore, a slight change in the PU activity of the CRN, which affects the channel availability probability, can have a significant impact on SUs with stringent latency requirements. The behavior happening at certain $q$ values, where the delay steeply increases caused by a minimal increase in $q$ is counter-intuitive, and it is investigated with the following example.

As an example, we have analyzed the behavior happening for $M=4$, and the channel availability probabilities of $q=0.5$ and $q=0.51$. Table II shows all the possible rate se-

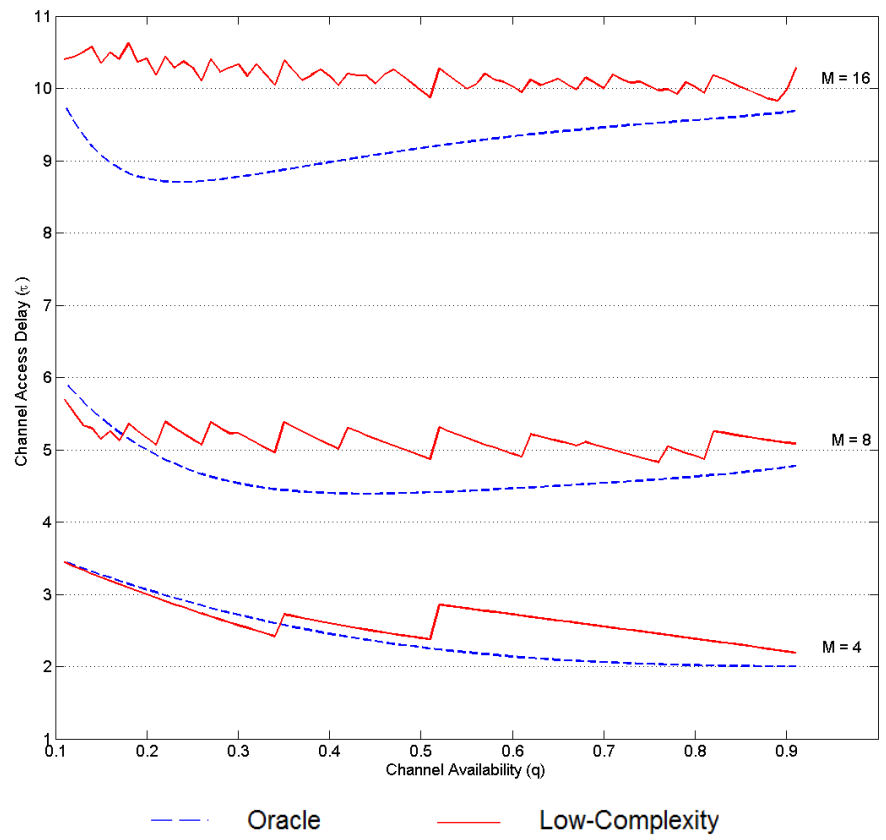

Fig. 3. Comparison of mean channel access delay for the Oracle and LowComplexity algorithms when varying channel availability $(q)$ in CRNs with number of channels $M=4, M=8$, and $M=16$. 
TABLE II

EXAmPle: Channel ACCess Delays for Possible Rate SeQuences

\begin{tabular}{c||c|c|c|c|c|c|c|c|c|c|c|c|c|c|c|c|}
\hline $\boldsymbol{a}_{\boldsymbol{s}_{\mathbf{1}}}$ & 0 & 0 & 0 & 0 & 0 & 0 & 0 & 0 & 1 & 1 & 1 & 1 & 1 & 1 & 1 & 1 \\
$\boldsymbol{a}_{\boldsymbol{s}_{2}}$ & 0 & 0 & 0 & 0 & 1 & 1 & 1 & 1 & 0 & 0 & 0 & 0 & 1 & 1 & 1 & 1 \\
$\boldsymbol{a}_{\boldsymbol{s}_{3}}$ & 0 & 0 & 1 & 1 & 0 & 0 & 1 & 1 & 0 & 0 & 1 & 1 & 0 & 0 & 1 & 1 \\
$\boldsymbol{a}_{\boldsymbol{s}_{\mathbf{4}}}$ & 0 & 1 & 0 & 1 & 0 & 1 & 0 & 1 & 0 & 1 & 0 & 1 & 0 & 1 & 0 & 1 \\
\hline \hline $\boldsymbol{m}_{\boldsymbol{\delta} \boldsymbol{\prime}}$ & 4 & 4 & 3 & 3 & 2 & 2 & 2 & 2 & 2 & 2 & 2 & 2 & 2 & 2 & 2 & 2 \\
\hline $\boldsymbol{m}_{\boldsymbol{\delta} \boldsymbol{\prime}}$ & 4 & 4 & 3 & 3 & 3 & 3 & 3 & 3 & 3 & 3 & 3 & 3 & 2 & 2 & 2 & 2 \\
\hline
\end{tabular}

quences and relative channel access delays determined by the following aggregate rate thresholds for the Low-Complexity algorithm: $\boldsymbol{\delta} \prime=[1.5,1.0,0.5,0] \delta \prime \prime=[1.53,1.02,0.51,0]$,for the two $q$ cases. For the case of $A_{2}=1$ (i.e., one channel available after exploring two channels), the Low-Complexity algorithm stopping rule will stop and transmit for $q=0.5$ (since $A_{2} \geqslant \delta_{2} \prime$ ) whereas it will continue for $q=0.51$ (since $\left.A_{2}<\delta_{2} \prime \prime\right)$. The change of the stopping rule for the case when an SU finds only one channel available after sensing two channels justifies the increase in the mean channel access delay and therefore the sawtooth behavior of the curves.

The analyzed behavior of the curves in Figure 3 indicates the need of accurate channel availability evaluations for singlerate SUs demanding very restricted delay variations.

\section{CONClusions And Future WORK}

In this paper, we have studied the delay and data rate performance of two optimal stopping algorithms designed for maximizing the throughput of multi-channel access SUs in a CRN. The simulation results give us guidelines on the feasibility of our solutions for mobile applications with stringent traffic requirements of delay, jitter and guaranteed data rate. We have seen how CRNs with higher number of channels seem to be more prone to support SUs with applications needing bounded delay and/or constant data rate. Also, both algorithms have shown to be more conservative than the optimum solution, stopping the channel exploration earlier and therefore having shorter channel access delays. Particularly, the computationally efficient Low-Complexity algorithm shows the best results for channel access delay, therefore making it the most suitable algorithm for mobile SUs. Finally, we have shown the coarsegrained predictability of channel access delays under varying PU activity conditions and the fine-grained instability of the same statistic, which suggest the feasibility of SU applications with latency requirements in the order of the coarse-grained values.

Motivated by the encouraging results found, we foresee the need of researching and developing multi-channel access algorithms based on the optimal stopping theory, which optimize other traffic specific requirements such as delay, jitter, and guaranteed data rate. These algorithms will permit the successful operation of QoS-based mobile applications as SUs in a CRN.

\section{REFERENCES}

[1] M. A. McHenry, "NSF Spectrum Occupancy Measurements Project Summary," Shared Spectrum Company, Tech. Report, Aug 2005.

[2] Part 22: Cognitive Wireless RAN Medium Access Control (MAC) and Physical Layer (PHY) Specifications: Policies and Procedures for operation in the TV Bands, IEEE 802.22 Working Group on Wireless Regional Area Networks, Jul. 12011.

[3] J. Wang, M. Ghosh, and K. Challapali, "Emerging cognitive radio applications: A survey," Communications Magazine, IEEE, vol. 49, no. 3, pp. $74-81$, Mar. 2011.

[4] T. Ferguson, Optimal stopping time and applications, http://www.math. ucla.edu/ $\sim$ tom/Stopping/Contents.html.

[5] H. Mahmoud, T. Yucek, and H. Arslan, "OFDM for cognitive radio: merits and challenges," Wireless Communications, IEEE, vol. 16, no. 2, pp. $6-15$, Apr. 2009.

[6] C. Cordeiro and M. Ghosh, Channel Bonding vs. Channel Aggregation: Facts and Faction, IEEE P802.22, https://mentor.ieee.org/802.22/dcn/06/ 22-06-0108-00-0000-bondingvs-aggregation.ppt.

[7] J. Jia, Q. Zhang, and X. Shen, "HC-MAC: A Hardware-Constrained Cognitive MAC for Efficient Spectrum Management," Selected Areas in Communications, IEEE Journal on, vol. 26, no. 1, pp. 106 -117, Jan. 2008.

[8] H. Jiang, L. Lai, R. Fan, and H. Poor, "Optimal selection of channel sensing order in cognitive radio," Wireless Communications, IEEE Transactions on, vol. 8, no. 1, pp. $297-307$, jan. 2009.

[9] H. T. Cheng and W. Zhuang, "Simple Channel Sensing Order in Cognitive Radio Networks," Selected Areas in Communications, IEEE Journal on, vol. 29, no. 4, pp. $676-688$, Apr. 2011.

[10] H. T. Cheng, H. Shan, and W. Zhuang, "Stopping Rule-Driven Channel Access in Multi-Channel Cognitive Radio Networks," in Communications (ICC), 2011 IEEE International Conference on, Jun. 2011, pp. 1 $-6$.

[11] L. Zappaterra, J. Gomes, A. Arora, and H.-A. Choi, "Resource Discovery Algorithms for Channel Aggregation in Cognitive Radio Networks," in Wireless Communications and Networking Conference (WCNC), 2013 IEEE, April 2013

[12] — "A Linear-Time Algorithm for Optimal Multi-Channel Access in Cognitive Radio Networks," in World of Wireless, Mobile and Multimedia Networks (WoWMoM), 2012 IEEE International Symposium on a (in review), June 2013.

[13] D. Niyato and E. Hossain, "Medium access control protocols for dynamic spectrum access in cognitive radio networks: A survey," Cognitive Radio Networks, pp. 179 -214, Dec. 2008.

[14] H. Urkowitz, "Energy detection of unknown deterministic signals," Proceedings of the IEEE, vol. 55, no. 4, pp. 523 - 531, april 1967.

[15] W. Gardner, "Signal interception: a unifying theoretical framework for feature detection," Communications, IEEE Transactions on, vol. 36, no. 8, pp. $897-906$, aug 1988.

[16] Z. Khan, J. LehtomŁki, L. DaSilva, and M. Latva-aho, "Autonomous sensing order selection strategies exploiting channel access information," Mobile Computing, IEEE Transactions on, vol. 12, no. 2, pp. $274-288$, feb. 2013

[17] T. Shu and M. Krunz, "Throughput-efficient sequential channel sensing and probing in cognitive radio networks under sensing errors," in Proceedings of the 15th annual international conference on Mobile computing and networking, ser. MobiCom '09. New York, NY, USA: ACM, 2009, pp. 37-48. 